\title{
Validation of Revised Baveno VI Criteria for Screening of Varices Needing Treatment in Children with Cirrhosis
}

This article was published in the following Dove Press journal:

Clinical and Experimental Gastroenterology

\author{
Tryambak Samanta $\mathbb{D}^{\prime}$ \\ Kalidas Biswas' \\ Saubhik Ghosh ${ }^{1}$ \\ Manoj Kumar Gupta' \\ Rajarshi Basu \\ Radheshyam Purkait ${ }^{2}$ \\ Abhisek Naskar ${ }^{2}$ \\ Shristi Butta ${ }^{3}$ \\ Debasis Das ${ }^{4}$ \\ Sutapa Ganguly ${ }^{5}$ \\ 'Department of Medical \\ Gastroenterology, Medical College, \\ Kolkata 73, India; ${ }^{2}$ Department of \\ Pediatrics, Nil Ratan Sircar Medical \\ College, Kolkata 14, India; ${ }^{3}$ Department \\ of Pathology, Nil Ratan Sircar Medical \\ College, Kolkata 14, India; ${ }^{4}$ Department \\ of Community Medicine, Medical \\ College, Kolkata 73, India; ${ }^{5}$ Department \\ of Pediatrics, KPC Medical College, \\ Kolkata, India
}

Correspondence: Manoj Kumar Gupta Senior Resident, Department of Medical Gastroenterology, Medical College, Kolkata, India

Email mkgupta.pintu@gmail.com
Background: Baveno VI criteria for screening varices needing treatment (VNT) have not yet been validated in an exclusive pediatric and adolescent set of the population, in whom baseline parameters differ in relation to adults. Therefore, our primary objective was to validate Baveno VI and its expanded form in children below 18 years of age. The secondary aim was to elicit whether any revision of the above criteria with a target of not missing more than $5 \%$ VNT could be more accurate for this age group.

Materials and Methods: The work was carried out in two medical institutes, over a span of 3 years. Consecutively enrolled patients below 18 years of age, with compensated cirrhosis confirmed by liver biopsy, were evaluated for related blood parameters, transient elastography (TE) and esophagogastroduodenoscopy.

Results: Out of the 33 recruited patients, five (15.15\%) met the criteria for VNT. The sensitivity, specificity, PPV and NPV of Baveno VI and Expanded Baveno VI were observed as $60 \%, 92.3 \%, 60 \%$ and $92.3 \%$, and $20 \%, 100 \%, 100 \%$ and $88 \%$, respectively. We found that the Revised Baveno VI criteria with TE $<19 \mathrm{kPa}$ and platelet count of $>175 \times 10^{9}$ cells $/ \mathrm{L}$, with sensitivity $100 \%$, specificity $79 \%$, PPV $45 \%$, NPV $100 \%$ and accuracy of $82 \%$, are more appropriate for this age group.

Conclusion: We propose that further multicentrer studies with a larger sample size should be conducted before incorporating Revised Baveno VI criteria for high-risk varices in patients below 18 years in future guidelines.

Keywords: transient elastography, platelet count, compensated advanced chronic liver disease, cACLD, high-risk varices

\section{Introduction}

Identification of varices needing treatment (VNT) is essential in the management of portal hypertension (PHT) in patients with cirrhosis. Although non-invasive parameters and scoring systems to detect VNT are still evolving, to date, the Baveno VI criteria have gained the most widespread acceptance for screening asymptomatic populations with compensated advanced chronic liver disease (cACLD), so as to avoid unnecessary esophagogastroduodenoscopy (EGD). ${ }^{1-4}$ The recommendations indicate that in patients with cACLD, those with a liver transient elastography (TE) value $<20 \mathrm{kPa}$ and a platelet count $>150 \times 10^{9}$ cells/L have a very low risk of VNT and, therefore, screening endoscopy need not be performed. This recommendation, when corroborated, was found to avoid between 10 and $30 \%$ of endoscopies with a low risk of mistakenly omitting $\mathrm{VNT}^{5-8}$ Nevertheless, considering the low 
prevalence of VNT $(<10 \%)$ in cACLD patients, Augustin et al formulated the Expanded Baveno VI criteria to maximize the number of avoided endoscopies while preserving a very low risk of incorrectly missing VNT $(<5 \%) .{ }^{9}$ The cut-off values of the expanded criteria were set to a platelet count $>110 \times 10^{9}$ cells $/ \mathrm{L}$ and $\mathrm{TE}<25 \mathrm{kPa}$, which were substantiated by two additional cohorts and further studies. ${ }^{10,11}$

However, neither the original nor the Expanded Baveno VI criteria nor the Baveno VI satellite symposium clearly states whether the above set of rules applies for pediatric and adolescent populations. ${ }^{4,9,12}$ This point is of utmost importance as the invasiveness of EGD as well as logistic constraints in resource-poor settings limit the routine use of the procedure in children. So, our primary objective is to validate both of the above criteria in a pediatric and adolescent subset. As different age groups below 18 years of age have distinct normative values of $\mathrm{TE},{ }^{13}$ and direct extrapolation of adult figures to the pediatric population is generally discouraged, we additionally presume that both criteria will fall short of the required precision in a young population, in contrast to adults. Therefore, our secondary aim is to find out whether any alteration of the above criteria would improve the screening of VNT in children, so that no more than $5 \%$ of cases are overlooked.

\section{Materials and Methods}

The study was conducted for a 3 year period concurrently in the Department of Pediatrics, Nil Ratan Sircar Medical College, Kolkata, India, where the pediatric population below 12 years was recruited, and the Department of Medical Gastroenterology, Medical College, Kolkata, India, where adolescents between 12 and 18 years were enlisted between December 2016 and November 2019. Prerequisite approvals were obtained from the Institutional Ethical Committees of both centers and informed consent was obtained from either the parent or primary care giver of each of the patients registered in the study. This study was performed in accordance with the Declaration of Helsinki.

Consecutive patients with compensated cirrhosis, confirmed by liver biopsy after inpatient admission, were recruited for further work-up. The parameters from baseline clinical and biochemical data which were specifically retrieved and analyzed were growth failure (height or weight below $2 \mathrm{SD}$ of the mean for age), ${ }^{14,15}$ platelet count, total serum bilirubin (TSB), serum aspartate aminotransferase (AST), serum albumin (S Alb) and international normalized ratio (INR). Pediatric End Stage Liver Disease (PELD) score ( $\leq 12$ years) and Model for End Stage Liver Disease- Sodium (MELD-Na) score $(\geq 12$ years) were calculated using additional baseline characteristics, as and when required. ${ }^{16,17}$

With proper consent, liver biopsy was carried out by a pathologist with a minimum post-residency experience of more than 3 years using a $\operatorname{Bard}^{\mathbb{B}}$ MaxCore ${ }^{\mathbb{R}}$ Disposable Core Biopsy Instrument $(16 \mathrm{G} \times 16 \mathrm{~cm})$ percutaneously under real-time image guidance, with strict adherence to prebiopsy preparations and peribiopsy management as per standard guidelines. ${ }^{18}$ Liver biopsy was postponed until 4 weeks after resolution of symptoms in those children presenting with features suggestive of acute hepatitis. For high INR, $>1.4$, due to cholestasis, administration of ageadjusted parenteral injection of vitamin $\mathrm{K}$ was performed before contemplating liver biopsy. ${ }^{19}$ After being satisfied about the adequacy of the size of the tissue biopsy sample, a histology report confirming cirrhosis needed to be established by a team of two pathologists, both at the level of consultant, who were not aware of the patient's clinical data.

TE (FibroScan ${ }^{\circledR}$; Echosens, Paris, France) examination was performed at the anterior axillary line (AAL) above the liver, with 10 valid measurements being obtained. Results were given as the median of these 10 measurements, and were deemed acceptable if the ratio of the interquartile range and median was $<30 \%$ and the success rate was $>60 \% .{ }^{13}$ The available FibroScan probes $(\mathrm{M} / \mathrm{S})$ offer three different depths of measurement: M, 25-65 $\mathrm{mm} ; \mathrm{S} 1,15-40 \mathrm{~mm}$; and $\mathrm{S} 2,20-50 \mathrm{~mm}$. The manufacturer's recommendations for probe choice, with cut-off values for chest circumference $(<45 \mathrm{~cm} \mathrm{S1}, 45-75 \mathrm{~cm}$ $\mathrm{S} 2,>75 \mathrm{~cm} \mathrm{M})$ and age $(<6$ years $\mathrm{S} 1,6-14$ years $\mathrm{S} 2,>14$ years $\mathrm{M}$ ), were primarily followed. The decision to defer this investigation by a maximum of 3 months, where applicable, was allowed, when the patient presented with acute hepatitis or bilirubinostasis. Measurements were preferably taken while the children/adolescents slept or were performed with conscious sedation, after a fast of atleast 30 minutes. ${ }^{20}$ The operator for TE in all cases was the first author.

EGD was carried out in all of the children using a pediatric endoscope (Olympus ${ }^{\circledR}$ GIF-XP180N, OD 5.5 $\mathrm{mm}$ ) in the Department of Medical Gastroenterology of Medical College, Kolkata. Esophageal varices, if any, were categorized into large $(>5 \mathrm{~mm})$ or small $(<5 \mathrm{~mm})$, 
and gastric varices, if any, were subdivided as per the Sarin classification. ${ }^{4,21}$ VNT were defined as the presence of large esophageal varices, gastric varices and varices with red color signs (RCS), either in isolation or in combination. ${ }^{4}$ Endoscopy was performed by any of the first three authors or under their supervision, and was documented only after $100 \%$ agreement in the observations between these authors, after reinspecting the videorecorded version of the procedure.

\section{Statistical Analysis}

Data, comprising statistical averages, distribution, dispersions and proportions, were compiled in Microsoft Excel ver. 2019 and analyzed using SAS software (SAS Institute, Cary, NC, USA). The mean \pm standard deviation (SD) were calculated and the chi-squared test and $Z$ tests were applied as and when applicable, at a significance level of $p<0.05$. As different age groups in our studied population have distinct sets of normative TE values, to maintain comparability, along with TE, deviation of values of TE from the reference, determined by positivity of the SD of TE (+SD of TE) to the nearest integer, was also interpreted. The biserial correlation coefficient $\left(r_{\mathrm{b}}\right)$ was measured between dependent dichotomous and independent variables. Binary logistic regression was used to predict the relationship of independent and dependent binary variables. Receiver operating characteristics (ROC) curves were formulated and the area under the ROC (AUROC) was calculated to evaluate the diagnostic abilities of parameters and their combination, as and when indicated. Cochran's $Q$ and McNemar's test were utilized to compare the different criteria applied to evaluate the non-invasive methods of screening VNT.

\section{Results}

Thirty-three (33) patients were prospectively enrolled after biopsy confirmation of cirrhosis in liver histology. Six $(18.18 \%), 11(33.33 \%)$ and $16(48.48 \%)$ subjects were in the age groups $0-6,6-12$ and $12-18$ years of age, respectively.

The etiologies noted were Wilson's disease (WD) $(n=8)$, autoimmune hepatitis $(\mathrm{AIH})(\mathrm{n}=5)$, secondary hemochromatosis $(n=4)$, extrahepatic biliary atresia (EHBA) $(n=4)$, chronic hepatitis $C(n=2)$, progressive familial intrahepatic cholestasis (PFIC) $(n=1)$, BuddChiari syndrome (BCS) $(n=1)$ and glycogen storage disease (GSD) type IV $(n=1)$, with an inconclusive diagnosis in seven cases. The presentations varied; however, 10 cases in this young subset of the population were detected incidentally. These included four patients with WD, with two each detected as part of an evaluation of hepatic involvement in neuropsychiatric WD and family screening. All four study subjects with secondary hemochromatosis and the two cases of chronic hepatitis $\mathrm{C}$ were chronic hemolytic anemia patients, who were referred only for high TE values from the hematology departments of the institutes, as part of a separate study protocol.

Five children (15.15\%) were identified as meeting the criteria for VNT, with the etiology being WD, AIH, BCS and GSD type IV in four of them. All five of these children had large esophageal varices, with gastroesophageal varices type 2 (GOV2) noted in one of these cases and RCS found in none. Table 1 shows the basic descriptive

Table I Descriptive Statistics of Baseline Characteristics of VNT, non-VNT and Total Cases

\begin{tabular}{|c|c|c|c|}
\hline Parameters & VNT $(n=5)$ & Non-VNT $(n=28)$ & Total $(n=33)$ \\
\hline Age (mean $\pm S D)$ (years) & $13.4 \pm 2.1$ & $10.83 \pm 1.03$ & $11.26 \pm 0.93$ \\
\hline $\operatorname{Sex}(M: F)$ & $1.5: 1$ & I.33:1 & I.35:1 \\
\hline PELD score $($ mean $\pm S D)(n=\mid 7)$ & $11.3(n=1)$ & $10.38 \pm 6.52(n=16)$ & $10.44 \pm 0.6 .33$ \\
\hline MELD-Na score $($ mean $\pm S D)(n=16)$ & $18 \pm 3.6(n=4)$ & $11.47 \pm 2.74(n=12)$ & $13.06 \pm 4.02$ \\
\hline Growth failure (n) & 1 & 16 & 17 \\
\hline $\mathrm{TE}(\mathrm{mean} \pm \mathrm{SD})(\mathrm{kPa})$ & $21.5 \pm 1.11$ & $16.5 \pm 0.54$ & $17.26 \pm 0.58$ \\
\hline$+\mathrm{SD}$ of TE $($ mean $\pm \mathrm{SD})$ & $15.8 \pm 1.24$ & $10.7 \pm 0.66$ & $11.48 \pm 0.67$ \\
\hline $\mathrm{PC}($ mean $\pm \mathrm{SD})\left(\times 10^{9}\right.$ cells $\left./ \mathrm{L}\right)$ & $108 \pm 17$ & $211 \pm 16$ & $195 \pm 15$ \\
\hline TSB $($ mean $\pm S D)(m g / d L)$ & $6.1 \pm 2.18$ & $4 \pm 0.7$ & $4.38 \pm 0.67$ \\
\hline S Alb (mean $\pm \mathrm{SD})(\mathrm{g} / \mathrm{dL})$ & $3.5 \pm 0.18$ & $3.36 \pm 0.05$ & $3.38 \pm 0.05$ \\
\hline AST (mean $\pm \mathrm{SD})(\mathrm{IU} / \mathrm{L})$ & $193 \pm 26.7$ & $168.5 \pm 14.8$ & $172.2 \pm 13.1$ \\
\hline INR (mean $\pm S D)$ & $1.46 \pm 0.02$ & $1.26 \pm 0.03$ & $1.28 \pm 0.03$ \\
\hline
\end{tabular}

Abbreviations: VNT, varices needing treatment; SD, standard deviation; PELD, Pediatric End Stage Liver Disease; MELD-Na, Model for End Stage Liver Disease-Sodium; TE, transient elastography; PC, platelet count; TSB, total serum bilirubin; S Alb, serum albumin; AST, serum aspartate aminotransferase; INR, international normalized ratio. 
Table 2 Values of the Statistical Parameters of the Selected Continuous Variables in Relation to Presence of VNT

\begin{tabular}{|l|l|l|l|l|l|l|l|}
\hline Statistical Parameters & TE & +SD of TE & PC & TSB & S Alb & AST & INR \\
\hline$r_{\mathrm{b}}$ & 0.83 & 0.79 & -0.65 & 0.26 & 0.23 & 0.17 & 0.26 \\
$P$ & 0.01 & 0.01 & 0.04 & 0.32 & 0.38 & 0.5 & 0.29 \\
AUROC & 0.91 & 0.89 & 0.9 & 0.71 & 0.62 & 0.60 \\
\hline
\end{tabular}

Abbreviations: VNT, varices needing treatment; $r_{b}$, biserial correlation coefficient; $p, p$ values of logistic regression in respect to variables; AUROC, area under the receiver operator characteristics curve; TE, transient elastography; SD, standard deviation; PC, platelet count; TSB, total serum bilirubin; S Alb, serum albumin; AST, serum aspartate aminotransferase; INR, international normalized ratio.

statistics of all the cases, along with comparative analysis of the group of patients with VNT and non-VNT.

At the outset, the biserial correlation coefficient $\left(r_{\mathrm{b}}\right)$, specific $p$ values of binary logistic regression and AUROC values of the individual continuous parameters in relation to the binary dependent outcome were computed, as tabulated in Table 2. Figure 1 compiles the ROC curves of the continuous independent potential variables. Growth failure was found to be non-significantly related to the presence of VNT ( $p=0.112)$. It is thus evident that TE, +SD of TE and platelet count were statistically significant predictors for having VNT.

The diagnostic accuracies of the Baveno VI and Expanded Baveno VI criteria were calculated (Table 3). In searching for better criteria for pediatric and adolescent populations, and inferring from the Youden index ( $J$ statistics) of ROC curves (Figure 1) and data in Table 2, TE and $+\mathrm{SD}$ of $\mathrm{TE}$ of $19 \mathrm{kPa}$ and 13, respectively, were found to be the best cut-off values in our studied age group. We now planned to fit the different sets of platelet counts with an interval of $25 \times 10^{9}$ cells/L $\left(125-250 \times 10^{9}\right.$ cells/L) after

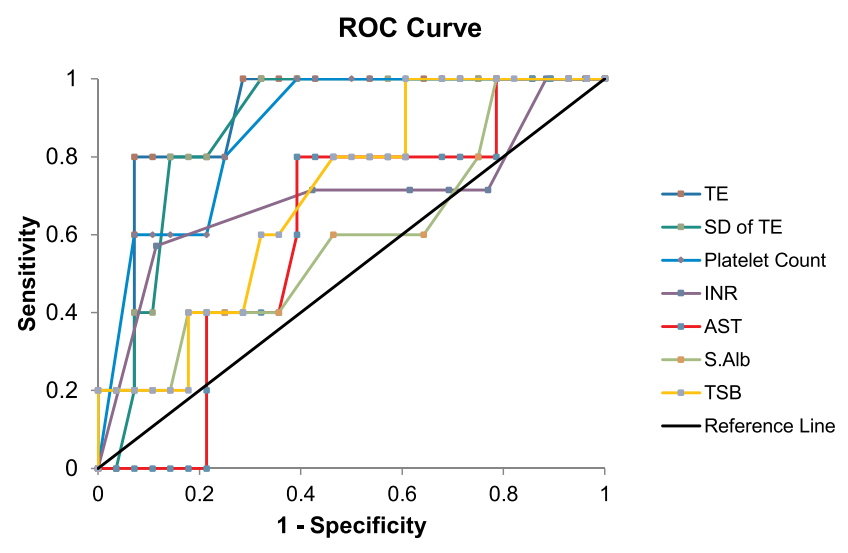

Figure I ROC curves of the dependent potential continuous variables in reference to non-invasive screening of varices needing treatment (VNT).

Abbreviations: TE, transient elastography; SD, standard deviation; INR, international normalized ratio; AST, aspartate amino transferase; S.Alb, serum albumin; TSB, total serum bilirubin. derivation from the ROC curves of the platelet count parameter.

Table 3 shows the diagnostic abilities of the different proposed criteria along with the existing criteria. Cochran's $Q$ test indicated that there was a significant difference $(p<0.001)$ between the criteria; therefore, McNemar's test was applied, which showed statistical significance between the criteria of Expanded Baveno VI with $\mathrm{TE}<19 \mathrm{kPa}$ or $+\mathrm{SD}$ of $\mathrm{TE}<13$ and platelet count $>125 \times 10^{9}$ cells $/ \mathrm{L}(p=0.04)$; TE $<19 \mathrm{kPa}$ or $+\mathrm{SD}$ of $\mathrm{TE}<13$ and platelet count $>150 \times 10^{9}$ cells $/ \mathrm{L}(p=0.02)$; TE $<19 \mathrm{kPa}$ or $+\mathrm{SD}$ of $\mathrm{TE}<13$ and platelet count $>175 \times 10^{9}$ cells $/ \mathrm{L}$ and above $(p=0.002)$, but not between Baveno VI and the proposed criteria. Figure 2 compares the ROCs of the existing and proposed criteria for non-invasive screening of VNT in this young subset of the population.

\section{Discussion}

Various authors have attempted to screen high-risk varices in the pediatric population. Gana et al and Adami et al initially noted that the best non-invasive predictors for large varices were platelet count, the clinical prediction rule and the risk score. They showed in a logistic regression model that the combination of the clinical prediction rule, the risk score and the platelet count/spleen size $Z$ score could be helpful in identifying cirrhotic children who may be eligible for endoscopy. ${ }^{22-24}$

In the following years, as a consequence of the increasing acceptance of TE, ${ }^{25}$ the Baveno VI consensus conference introduced a non-invasive screening method for screening VNT in the management of patients with cACLD primarily in the adult population. The original Baveno VI criteria, based on simple analytical TE values, were well validated in subsequent studies, ${ }^{5-8}$ but were also criticized as being conservative; endoscopies were spared in modest numbers, and most strikingly $40 \%$ of unwanted endoscopies still needed to be carried out. In a multicenter study, Protopapas et $\mathrm{al}^{11}$ further noted that the absence of the Baveno VI criteria 
Table 3 Diagnostic Abilities of Established and Proposed Criteria for Screening VNT in Children†

\begin{tabular}{|c|c|c|c|c|c|c|c|}
\hline Criteria $\ddagger$ & Sn & Sp & PPV & NPV & LR+ & LR- & Overall Accuracy \\
\hline Baveno VI & 0.6 & 0.92 & 0.6 & 0.93 & 8.4 & 0.43 & 0.87 \\
\hline Expanded Baveno VI & 0.2 & 1 & 1 & 0.88 & NA & 0.80 & 0.87 \\
\hline TE (19)+PC (125) & 0.6 & 0.86 & 0.43 & 0.93 & 4.2 & 0.47 & 0.81 \\
\hline TE (19)+PC(150) & 0.8 & 0.86 & 0.5 & 0.96 & 5.6 & 0.23 & 0.84 \\
\hline TE (19)+PC (I75) & I & 0.79 & 0.45 & I & 4.67 & 0 & 0.82 \\
\hline TE (19)+PC (200) & I & 0.79 & 0.45 & I & 4.67 & 0 & 0.82 \\
\hline TE (19)+PC (225) & I & 0.79 & 0.45 & I & 4.67 & 0 & 0.82 \\
\hline TE (19)+PC (250) & I & 0.79 & 0.45 & I & 4.67 & 0 & 0.82 \\
\hline$+\mathrm{SD}(13)+\mathrm{PC}(125)$ & 0.6 & 0.86 & 0.43 & 0.93 & 4.2 & 0.47 & 0.81 \\
\hline$+S D(13)+P C(150)$ & 0.8 & 0.86 & 0.5 & 0.96 & 5.6 & 0.23 & 0.84 \\
\hline$+S D(13)+P C(175)$ & 1 & 0.79 & 0.45 & I & 4.67 & 0 & 0.82 \\
\hline$+S D(13)+P C(200)$ & I & 0.79 & 0.45 & I & 4.67 & 0 & 0.82 \\
\hline +SD (13)+PC (225) & I & 0.79 & 0.45 & I & 4.67 & 0 & 0.82 \\
\hline$+S D(13)+P C(250)$ & I & 0.79 & 0.45 & I & 4.67 & 0 & 0.83 \\
\hline
\end{tabular}

Notes: $\nmid$ Values in decimals. $\ddagger$ Values in parentheses for TE, SD and PC denote the respective values, with the unit of measurement being $\mathrm{kPa}$ for $\mathrm{TE}$ and $\times 10^{9}$ cells/L for $\mathrm{PC}$. Abbreviations: VNT, varices needing treatment; TE, transient elastography; SD, standard deviation of TE; PC, platelet count; Sn, sensitivity; Sp, specificity; PPV, positive predictive value; NPV, negative predictive value; $L R+$, positive likelihood ratio; $L R-$, negative likelihood ratio; NA, not applicable.

predicted the detection of VNT with $100 \%$ sensitivity, $15 \%$ specificity, $23 \%$ positive predictive value (PPV) and $100 \%$ negative predictive value (NPV). While validating the Baveno VI criteria, probably for the first time in an exclusively young population, we observed that although the specificity and NPV of the criteria were high (92.3\%), the PPV was low (60\%) and, most strikingly, two of the five VNT were missed (sensitivity 60\%), which is unacceptable in a clinical setting.

As previously mentioned, consequent to the Baveno VI criteria, there have been many studies proposing alterations to the criteria, in order to maximize the efficacy of non-invasive tools used to detect VNT. The most significant among these is a study ${ }^{9}$ that proposed

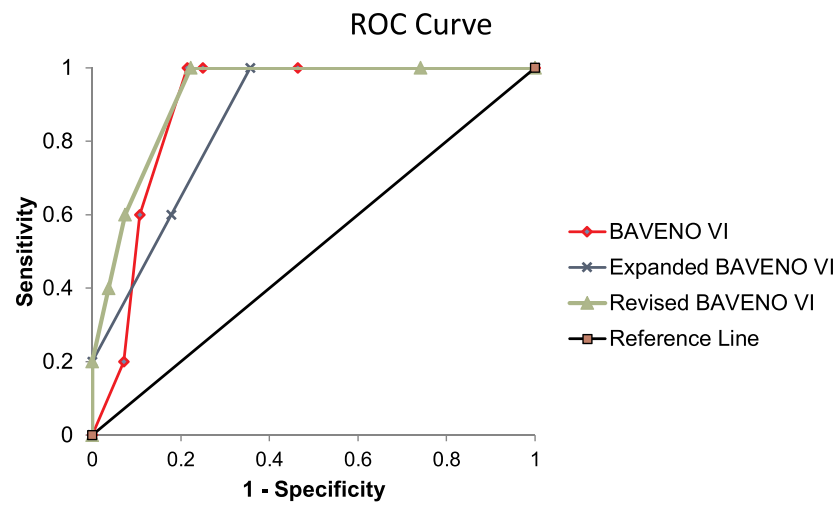

Figure 2 ROCs of the existing and proposed criteria for non-invasive screening of varices needing treatment (VNT) in a population aged less than 18 years.

Notes: Fitted AUROCs of Baveno VI, Expanded Baveno VI and Revised Baveno VI are $0.9,0.85$ and 0.94 , respectively. expanding the Baveno VI criteria to include patients with a platelet count $>110 \times 10^{9}$ cells/L and TE $<25$ $\mathrm{kPa}$. This study reported that the expanded form of criteria resulted in a significant decrease in the number of EGDs while keeping within the criteria a meager percentage of patients with missed VNT. However, in our study, these criteria failed to show diagnostic ability (Table 3). This can likely be explained by the fact that normative TE values in the pediatric age group and adolescents are in the lower range, so the higher cutoff is unlikely to be applicable.

Inspired by the above paradigm, we focused on précising the cut-offs of relevant parameters for the specified age group to find a better version of the original criteria for the studied population. Therefore, we investigated every single curve point of the ROC of all the potential variables and, as expected, TE of $19 \mathrm{kPa}$ and $+\mathrm{SD}$ of TE of 13 were the two factors that correlated most closely with VNT, with sensitivity, specificity, PPV and NPV of the same measurements of $60 \%, 78.5 \%, 40 \%$ and $95.6 \%$, respectively. In an effort to gain better precision of the criteria, the platelet count parameter was added to the above values of TE and $+\mathrm{SD}$ of TE, as it was the only variable other than $\mathrm{TE}$ and $+\mathrm{SD}$ of $\mathrm{TE}$ that correlated significantly $\left(r_{\mathrm{b}}=-0.65\right)$ with VNT. As is evident from Table 2, platelet counts of $175 \times 10^{9}$ cells/L and above, coupled with the above-mentioned cut-offs of TE or +SD of TE, was judged to be a more diagnostically accurate cut-off for differentiating VNT from non-VNT in 
juveniles. However, at the bedside, as SD calculation is obviously cumbersome, we considered TE $<19 \mathrm{kPa}$ with platelet count $>175 \times 10^{9}$ cells/ $\mathrm{L}$ as the best criteria for our study population.

Prima facie, our study's strength lies in the fact that it was probably the first attempt to find a better diagnostically related alternative tool compared to the Baveno VI criteria for non-invasive screening of VNT exclusively for children and adolescents. To the best of our knowledge, publications even in the recent past in regard to screening high-risk varices with PHT in children have not dealt with Baveno VI criteria. ${ }^{26-30}$

However, our foremost weakness was the scarcity of the cACLD cases below 18 years, thus restricting our sample size, which meant that the plan had to be earmarked as a pilot study only. Six children (18.18\%), comprising four infants with EHBA and one toddler with PFIC, continued to have high TSB, even after delivering available treatment, at 3 months of enrollment, thus acting as an effect modifier of TE values. More than moderate transaminitis ( $>5$ times of upper limit of normal) was present in two cases, despite serial biochemical monitoring up to 12 weeks following enlisting, which could also have given spuriously high values of $\mathrm{TE}$ in those specific patients.

\section{Conclusions}

Nonetheless, in parallel with validation, we have been able to document the gaps in theBaveno VI criteria in a young population. Extrapolating from our study results, we are not in favor of the use of Expanded Baveno VI criteria in this age group. Although we are proposing Revised Baveno VI criteria on the basis of TE and platelet count, we will not yet be overzealous in recommending its use on a routine basis in patients under 18 years of age. However, our study could provide the basis for formulating future research hypotheses, which could lead to precise revision of the Baveno VI criteria and/or detection of better noninvasive methods for screening for VNT in children and adolescents with compensated cirrhosis.

\section{Author Details}

Authors were formerly attached as Assistant Professor: Sutapa Ganguly, and Professor: Sutapa Ganguly, in the Department of Pediatrics, Nil Ratan Sircar Medical College, Kolkata, India.

\section{Acknowledgment}

The authors acknowledge the assistance of Dr Anup Sarkar, Senior Resident, Department of Medical Gastroenterology, Medical College, Kolkata-73, India, for his contribution to the performance of procedures.

\section{Author Contributions}

This is to state that all authors made substantial contributions to conception and design, acquisition of data, or analysis and interpretation of data; took part in drafting the article or revising it critically for important intellectual content; agreed on the journal to which the article will be submitted; gave final approval of the version to be published; and agree to be accountable for all aspects of the work.

\section{Funding}

The investigations were carried out in house using the existing resources of the institutions, which are funded by the Department of Health and Family Welfare, Government of West Bengal, India.

\section{Disclosure}

The authors report no conflicts of interest for this work. This study received no special or exclusive financial support.

\section{References}

1. Abd-Elsalam S, Habba E, Elkhalawany W, et al. Correlation of platelets count with endoscopic findings in a cohort of Egyptian patients with liver cirrhosis [published correction appears in Medicine (Baltimore). 2016 Jul 18;95(28):e0916]. Medicine. 2016;95(23): e3853. doi:10.1097/MD.0000000000003853

2. Wasfy E, Elkassas G, Elnawasany S, et al. Predicting esophageal varices in cirrhotic hepatitis $\mathrm{C}$ virus patients using noninvasive measurement of insulin resistance variables. Endocr Metab Immune Disord Drug Targets. 2018;18(6):573-580. doi:10.2174/18715303186661808 03120428

3. Hanafy AS, Badawi R, Basha MAA, et al. A novel scoring system for prediction of esophageal varices in critically ill patients. Clin Exp Gastroenterol. 2017;10:315-325. doi:10.2147/CEG.S144700

4. de Franchis R; Baveno VI Faculty. Expanding consensus in portal hypertension: report of the Baveno VI Consensus Workshop: stratifying risk and individualizing care for portal hypertension. $J$ Hepatol. 2015;63:743-752. doi:10.1016/j.jhep.2015.05.022

5. Maurice JB, Brodkin E, Arnold F, et al. Validation of the Baveno VI criteria to identify low risk cirrhotic patients not requiring endoscopic surveillance for varices. $J$ Hepatol. 2016;65:899-905. doi:10.1016/j. jhep.2016.06.021

6. Perazzo H, Fernandes FF, Castro Filho EC, et al. Points to be considered when using transient elastography for diagnosis of portal hypertension according to the Baveno's VI consensus. J Hepatol. 2015;63:1048-1049. doi:10.1016/j.jhep.2015.06.036 
7. Augustin S, Pons M, Genesca J. Validating the Baveno VI recommendations for screening varices. J Hepatol. 2017;66:459-460. doi:10.1016/j.jhep.2016.09.027

8. Marot A, Trepo E, Doerig C, et al. Liver stiffness and platelet count for identifying patients with compensated liver disease at low risk of variceal bleeding. Liver Int. 2017;37:707-716. doi:10.1111/liv.13318

9. Augustin S, Pons M, Maurice JB, et al. Expanding the Baveno VI criteria for the screening of varices in patients with compensated advanced chronic liver disease. Hepatology. 2017;66:1980-1988. doi:10.1002/hep.29363

10. Bae J, Sinn DH, Kang W, et al. Validation of the Baveno VI and the expanded Baveno VI criteria to identify patients who could avoid screening endoscopy. Liver Int. 2018;38(8):1442-1448. doi:10.1111/liv.13732

11. Protopapas AA, Mylopoulou T, Papadopoulos VP, et al. Validating and expanding the Baveno VI criteria for esophageal varices in patients with advanced liver disease: a multicenter study. Ann Gastroenterol. 2020;33(1):87-94.

12. Shneider BL, de Ville de Goyet J, Leung DH, et al. Primary prophylaxis of variceal bleeding in children and the role of MesoRex Bypass: summary of the Baveno VI Pediatric Satellite Symposium. Hepatology. 2016;63(4):1368-1380. doi:10.1002/hep.28153

13. Engelmann G, Gebhardt C, Wenning D, et al. Feasibility study and control values of transient elastography in healthy children. Eur $J$ Pediatr. 2012;171:353-360. doi:10.1007/s00431-011-1558-7

14. WHO Multicentre Growth Reference Study Group. WHO Child Growth Standards based on length/height, weight and age. Acta Paediatr Suppl. 2006;450:76-85.

15. Khadilkar V, Yadav S, Agrawal KK, et al.; Indian Academy of Pediatrics Growth Charts Committee. Revised IAP growth charts for height, weight and body mass index for 5- to 18-year-old Indian children. Indian Pediatr. 2015;52(1):47-55. doi:10.1007/s13312-015-0566-5.

16. McDiarmid SV, Merion RM, Dykstra DM, et al. Selection of pediatric candidates under the PELD system. Liver Transpl. 2004;10(10 Suppl 2):S23-S30. doi:10.1002/1t.20272

17. Machicao VI. Model for end-stage liver disease-sodium score: the evolution in the prioritization of liver transplantation. Clin Liver Dis. 2017;21(2):275-287. doi:10.1016/j.cld.2016.12.014

18. Rockey DC, Caldwell SH, Goodman ZD, et al.; American Association for the Study of Liver Diseases. Liver biopsy. Hepatology. 2009;49(3):1017-1044. doi:10.1002/hep.22742.

19. Curry MP, Jeffers LJ. Laboratory tests, noninvasive markers of fibrosis, liver biopsy, and laparoscopy. In: Schiff ER, Maddrey WC, Reddy KR, editors. Schiff's Diseases of the Liver. 12th ed. Chichester: Wiley Blackwell; 2017:17-37
20. Goldschmidt I, Streckenbach C, Dingemann C, et al. Application and limitations of transient liver elastography in children. $J$ Pediatr Gastroenterol Nutr. 2013;57(1):109-113. doi:10.1097/MPG.0b013e3 $1829206 \mathrm{a} 0$

21. Sarin SK, Kumar A. Gastric varices: profile, classification, and management. Am J Gastroenterol. 1989;84(10):1244-1249.

22. Gana JC, Turner D, Mieli-Vergani G, et al. A clinical prediction rule and platelet count predict esophageal varices in children. Gastroenterology. 2011;141(6):2009-2016. doi:10.1053/j.gastro.20 11.08 .049

23. Gana JC, Turner D, Roberts EA, et al. Derivation of a clinical prediction rule for the noninvasive diagnosis of varices in children [published correction appears in J Pediatr Gastroenterol Nutr. 2013 Apr;56(4):459]. J Pediatr Gastroenterol Nutr. 2010;50(2):188-193. doi:10.1097/MPG.0b013e3181b64437

24. Adami MR, Kieling CO, Schwengber FP, et al. Noninvasive methods of predicting large esophageal varices in children with intrahepatic portal hypertension. J Pediatr Gastroenterol Nutr. 2018;66(3):442446. doi:10.1097/MPG.0000000000001841

25. Castera L, Forns X, Alberti A. Non-invasive evaluation of liver fibrosis using transient elastography. J Hepatol. 2008;48(5):835847. doi:10.1016/j.jhep.2008.02.008

26. Bozic MA, Puri K, Molleston JP. Screening and prophylaxis for varices in children with liver disease. Curr Gastroenterol Rep. 2015;17(7):27. doi:10.1007/s11894-015-0450-4

27. Duché M, Ducot B, Ackermann O, et al. Portal hypertension in children: high-risk varices, primary prophylaxis and consequences of bleeding. J Hepatol. 2017;66(2):320-327. doi:10.1016/j.jhep.20 16.09 .006

28. Pimenta JR, Ferreira AR, Fagundes ED, et al. Factors associated with bleeding secondary to rupture of esophageal varices in children and adolescents with cirrhosis. J Pediatr Gastroenterol Nutr. 2017;64(2): e44-e48. doi:10.1097/MPG.0000000000001362

29. Sutton H, Dhawan A, Grammatikopoulos T. Non-invasive markers of portal hypertension: appraisal of adult experience and potential utilisation in children. J Pediatr Gastroenterol Nutr. 2018;66(4):559-569. doi:10.1097/MPG.0000000000001882

30. Henkel SAF, Squires JE. New tools for screening children with portal hypertension. J Pediatr Gastroenterol Nutr. 2019;69(6):639-640. doi:10.1097/MPG.0000000000002492
Clinical and Experimental Gastroenterology

\section{Publish your work in this journal}

Clinical and Experimental Gastroenterology is an international, peerreviewed, open access, online journal publishing original research, reports, editorials, reviews and commentaries on all aspects of gastroenterology in the clinic and laboratory. This journal is indexed on American Chemical Society's Chemical Abstracts Service (CAS).
The manuscript management system is completely online and includes a very quick and fair peer-review system, which is all easy to use. Visit http://www.dovepress.com/testimonials.php to read real quotes from published authors. 